\title{
Structure of traveling wave solutions for some nonlinear models via modified mathematical method
}

https://doi.org/10.1515/phys-2018-0107

Received Jul 22, 2018; accepted Oct 19, 2018

Abstract: We have employed the $\exp (-\varphi(\xi))$-expansion method to derive traveling waves solutions of breaking solition (BS), Zakharov-Kuznetsov-Burgers (ZKB), AblowitzKaup-Newell-Segur (AKNS) water wave, Unstable nonlinear Schrödinger (UNLS) and Dodd-Bullough-Mikhailov (DBM) equations. These models have valuable applications in mathematical physics. The results of the constructed model, along with some graphical representations provide the basic knowlegde about these models. The derived results have various applications in applied science.

Keywords: Breaking soliton equation, Three-dimensional ZKB equation, Ablowitz-Kaup-Newell-Segur equation, Unstable nonlinear NLS, Dodd-Bullough-Mikhailov equation (DBME), Mathematical method

PACS: 02.30.Jr, 04.30.Nk, 05.45.Yv

\section{Introduction}

Partial differential equations (PDEs) have been measured with great significance due to its variety of applications in physics, applied mathematics and engineering. PDEs can be used to describe a wide variety of phenomena such as sound, heat, electrostatics, electrodynamics, fluids dynamics, elasticity and quantum mechanics. These seemingly distinct physical phenomena can be formalized sim- ilarly in terms of PDEs. Due to its broad/various applications and important mathematical properties, many methods have been presented to study in different aspects related with the solutions and physical phenomena of nonlinear wave equations. Hence, penetrating and constructing exact traveling wave solutions for nonlinear differential equations is a modern research area. Numerous effective methods were discussed to obtain solutions of nonlinear wave system of equations in different aspects [1-10].

Recently, many new powerful methods have been proposed for finding the exact traveling waves solution of nonlinear evolution equations such as: the inverse scattering transform method, the homogeneous balance method, modified simple equation method, modified extended direct algebraic method, the tanhsech method and the extended tanhcoth method, the soliton ansatz method [11-20] and many more [21-40]. In previous studies the authors [23-29] applied, auxiliary equation, extended mapping, modified simple equation, modified extended and $\frac{G}{G^{\prime}}$ expansion methods on breaking solition (BS), Zakharov-Kuznetsov-Burgers (ZKB), AblowitzKaup-Newell-Segur (AKNS) water wave, unstable nonlinear Schrödinger (UNLS) and Dodd-Bullough-Mikhailov (DBM) equations, respectively. But here our aim is to investigate the novel exact and solitary wave solutions of these models by employing $\exp (-\varphi(\xi))$-expansion method.

The description of method is given in Section 2. In section 3, we apply the present method on selective models. Results and discussion are presented in Section 4. Finally, the Conclusions are given in Section 5.

\section{Description of the method}

^Corresponding Author: Aly R. Seadawy: Mathematics Department, Faculty of Science, Taibah University, Al-Madinah AlMunawarah, Saudi Arabia; Mathematics Department, Faculty of Science, Beni-Suef University, Egypt; Email: Aly742001@yahoo.com Dianchen Lu: Faculty of Science, Jiangsu University, Zhenjiang, Jiangsu 212013, P. R. China

Asghar Ali: Faculty of Science, Jiangsu University, Zhenjiang, Jiangsu 212013, P. R. China; Department of Mathematics, University of Education, Multan Campus, Pakistan

๑ Open Access. () 2018 D. Lu et al., published by De Gruyter.
Consider PDE in the form

$$
G\left(v, v_{t}, v_{x}, v_{y}, v_{z}, v_{x x}, v_{y y}, v_{z z}, \ldots\right)=0,
$$

where $G$ is a polynomial function in $v(x, y, z, t)$. Suppose,

$$
v(x, y, z, t)=V(\xi), \quad \xi=x+y+z-\omega t,
$$


Put (2) in (1),

$$
Q\left(V, V^{\prime}, V^{\prime \prime}, V^{\prime \prime \prime}, \ldots\right)=0,
$$

where $Q$ is a polynomial in $V$

Let (3) solution,

$$
V=A_{m}(\exp (-\varphi(\xi)))^{m}+\ldots, A_{m} \neq 0,
$$

where $\varphi(\xi)$ gratifies,

$$
\varphi^{\prime}(\xi)=\exp (-\varphi(\xi))+\mu_{1} \exp (\varphi(\xi))+\lambda_{1},
$$

Case 1. $\lambda_{1}^{2}-4 \mu_{1}>0, \mu_{1} \neq 0$ then (5) has solution,

$$
\varphi=\ln \left(\frac{-\sqrt{\lambda_{1}^{2}-4 \mu_{1}} \tanh \left(\frac{\sqrt{\lambda_{1}^{2}-4 \mu_{1}}}{2}\left(\xi+\xi_{0}\right)\right)-\lambda_{1}}{2 \mu_{1}}\right)
$$

Case 2. $\lambda_{1}^{2}-4 \mu_{1}>0, \quad \mu_{1}=0$ then (5) has solution,

$$
\varphi=-\ln \left(\frac{\lambda_{1}}{\exp \left(\lambda_{1}\left(\xi+\xi_{0}\right)\right)-1}\right)
$$

Case 3. $\lambda_{1}^{2}-4 \mu_{1}=0, \mu_{1} \neq 0, \lambda_{1} \neq 0$, (5) has solution,

$$
\varphi=\ln \left(-\frac{2\left(\lambda_{1}\left(\xi+\xi_{0}\right)+2\right)}{\lambda_{1}^{2}\left(\xi+\xi_{0}\right)}\right)
$$

Case 4. $\lambda_{1}^{2}-4 \mu_{1}=0, \mu_{1}=0, \lambda_{1}=0$, (5) has solution,

$$
\varphi=\ln \left(\xi+\xi_{0}\right)
$$

Case 5. $\lambda_{1}^{2}-4 \mu_{1}<0$, (5) has the following solution

$$
=\ln \left(\frac{\sqrt{4 \mu_{1}-\lambda_{1}^{2}} \tan \left(\frac{\sqrt{4 \mu_{1}-\lambda_{1}^{2}}}{2}\left(\xi+\xi_{0}\right)\right)-\lambda_{1}}{2 \mu_{1}}\right)
$$

Substituting (4) with (5) in (3), adjusting coefficients of $\exp (-m \varphi(\xi)), \mathrm{m}=0,1,2,3, \ldots$ equal to zero, we achieve numerous equations that can be solved with use of Mathematica.

Putting all values of parameters with solution of (5) in (4), we obtain solution of (1)

\section{Applications}

\section{1 (3+1)-dimensional BS equation}

Consider general form of BS equation in [23]

$$
v_{x t}+\alpha_{1} v_{x}\left(v_{x y}+v_{x z}\right)+\alpha_{2} v_{x x}\left(v_{y}+v_{z}\right)
$$

$$
+\alpha_{3}\left(v_{x x x y}+v_{x x x z}\right)=0,
$$

Suppose the transformations,

$$
v(x, y, z, t)=V(\xi), \quad \xi=x+y+z-\omega t,
$$

Put (12) in (11), after integrating,

$$
-\omega V^{\prime}+\left(\alpha_{1}+\alpha_{2}\right)\left(V^{\prime}\right)^{2}+2 \alpha_{3} V^{\prime \prime \prime}=0
$$

Let (13) has solution,

$$
V=A_{0}+A_{1} \exp (-\varphi(\xi))
$$

Substituting (14) with (5) in (13), we attained several equations

$$
A_{0}=A_{0}, \quad A_{1}=\frac{12 \alpha_{3}}{\left(\alpha_{1}+\alpha_{2}\right)}, \quad \omega=\left(2 \lambda_{1}^{2}-8 \mu_{1}\right) \alpha_{3}
$$

Then (14) becomes,

$$
V=A_{0}+\frac{12 \alpha_{3}}{\left(\alpha_{1}+\alpha_{2}\right)} \exp (-\varphi(\xi))
$$

Case 1. $\lambda_{1}^{2}-4 \mu_{1}>0, \quad \mu_{1} \neq 0$

$$
\begin{aligned}
& V_{1}=A_{0} \\
& +\frac{24 \alpha_{3} \mu_{1}}{\left(\alpha_{1}+\alpha_{2}\right)\left(-\sqrt{\lambda_{1}^{2}-4 \mu_{1}} \tanh \left(\frac{\sqrt{\lambda_{1}^{2}-4 \mu_{1}}}{2}\left(\xi+\xi_{0}\right)\right)-\lambda_{1}\right)}
\end{aligned}
$$

Case 2. $\lambda_{1}^{2}-4 \mu_{1}>0, \mu_{1}=0$,

$$
V_{2}=A_{0}+\frac{1}{\left(\alpha_{1}+\alpha_{2}\right)}\left(\frac{12 \alpha_{3} \lambda_{1}}{\exp \left(\lambda_{1}\left(\xi+\xi_{0}\right)\right)-1}\right)
$$

Case 3. $\lambda_{1}^{2}-4 \mu_{1}=0, \mu_{1} \neq 0, \lambda_{1} \neq 0$,

$$
V_{3}=A_{0}-\frac{1}{\left(\alpha_{1}+\alpha_{2}\right)}\left(\frac{6 \alpha_{3} \lambda_{1}^{2}\left(\xi+\xi_{0}\right)}{\left(\lambda_{1}\left(\xi+\xi_{0}\right)+2\right)}\right)
$$

Case 4. $\lambda_{1}^{2}-4 \mu_{1}=0, \mu_{1}=0, \lambda_{1}=0$,

$$
V_{4}=A_{0}+\frac{12 \alpha_{3}}{\left(\alpha_{1}+\alpha_{2}\right)\left(\xi+\xi_{0}\right)}
$$

Case 5. If $\lambda_{1}^{2}-4 \mu_{1}<0$,

$$
\begin{aligned}
& V_{5}=A_{0} \\
& +\frac{24 \alpha_{3} \mu_{1}}{\left(\alpha_{1}+\alpha_{2}\right)\left(\sqrt{4 \mu_{1}-\lambda_{1}^{2}} \tan \left(\frac{\sqrt{4 \mu_{1}-\lambda_{1}^{2}}}{2}\left(\xi+\xi_{0}\right)\right)-\lambda_{1}\right)}
\end{aligned}
$$




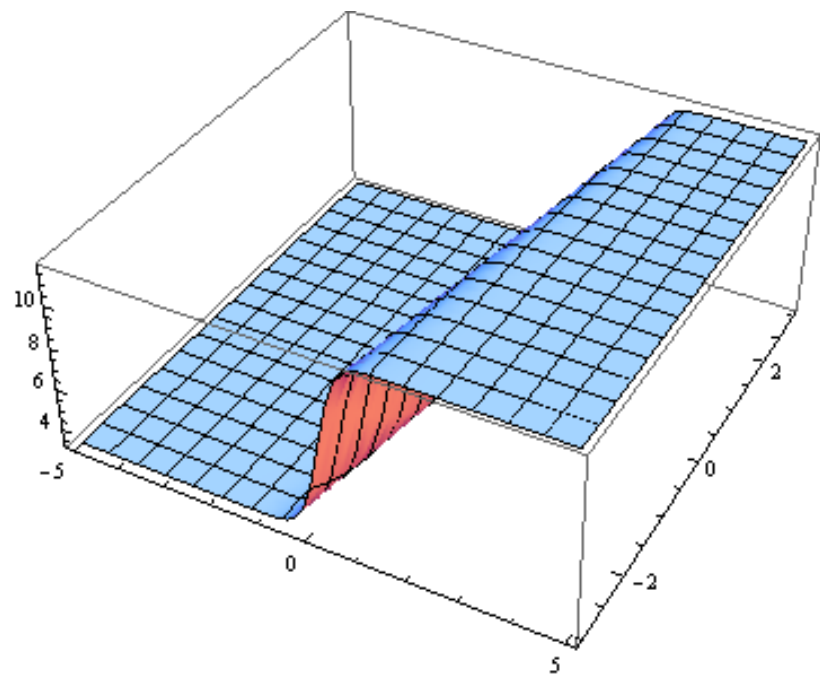

(a)

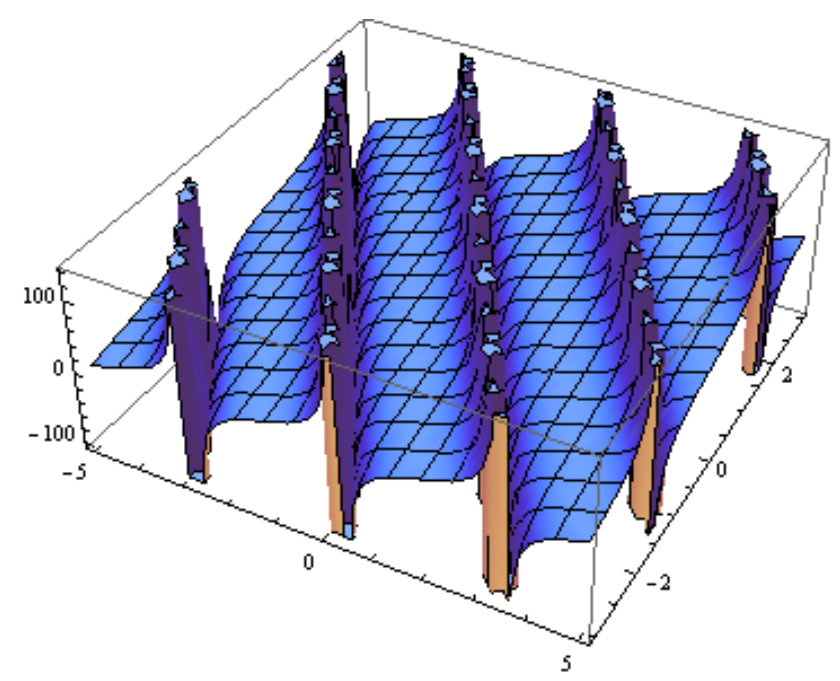

(b)

Figure 1: Solitary waves of solutions (17), (21) on (a), (b) with: $A_{0}=1.5, \lambda_{1}=2, \mu_{1}=0.7, \alpha_{1}=\alpha_{2}=1.0, \alpha_{3}=-1.0, \epsilon=0.5$ and $A_{0}=1.6$, $\lambda_{1}=2, \mu_{1}=2, \alpha_{1}=\alpha_{2}=1.1, \alpha_{3}=-3, \epsilon=.6$ respectively.

\section{$3.2(3+1)$-dimensional ZKB equation}

The general form of three-dimensional ZakharovKuznetsov-Burgers equation [24, 25],

$$
v_{t}+\beta_{1} v v_{x}+\beta_{2} v_{x x x}+\beta_{3}\left(v_{y y x}+v_{z z x}\right)+\beta_{4} v_{x x}=0
$$

Let the transformations,

$$
v(x, t)=V(\xi), \quad \xi=k x+l y+m z-\omega t,
$$

Put (23) in (22),

$$
\begin{aligned}
& -\omega V^{\prime}+\beta_{1} k V V^{\prime}+\beta_{4} k^{2} V^{\prime \prime} \\
& +\left(\beta_{2} k^{3}+\beta_{3} k l^{2}+\beta_{3} k m^{2}\right) V^{\prime \prime \prime}=0 .
\end{aligned}
$$

Let (24) has solution form of (14). Substituting (14) with (5) into Eq.(24), after solving we have,

$$
A_{0}=\frac{\beta_{4} \lambda_{1} k^{2}+\omega}{\beta_{1} k} \quad A_{1}=\frac{2 \beta_{4} k}{\beta_{1}} \quad m= \pm \sqrt{\frac{-k^{2} \beta_{2}}{\beta_{3}}-l^{2}},
$$

Thus (14) can be written as:

$$
V=\frac{\beta_{4} \lambda_{1} k^{2}+\omega}{\beta_{1} k}+\frac{2 \beta_{4} k}{\beta_{1}} \exp (-\varphi(\xi))
$$

Case 1. $\lambda_{1}^{2}-4 \mu_{1}>0, \mu_{1} \neq 0$

$$
\begin{aligned}
V_{6} & =\frac{\beta_{4} \lambda_{1} k^{2}+\omega}{\beta_{1} k} \\
& +\frac{4 k \beta_{4} \mu_{1}}{\beta_{1}\left(-\sqrt{\lambda_{1}^{2}-4 \mu_{1}} \tanh \left(\frac{\sqrt{\lambda_{1}^{2}-4 \mu_{1}}}{2}\left(\xi+\xi_{0}\right)\right)-\lambda_{1}\right)}, \\
& k>l, \quad \beta_{3}>0, \quad \beta_{2}<0 .
\end{aligned}
$$

Case 2. $\lambda_{1}^{2}-4 \mu_{1}>0, \mu_{1}=0$,

$$
\begin{aligned}
V_{7} & =\frac{\beta_{4} \lambda_{1} k^{2}+\omega}{\beta_{1} k}+\left(\frac{2 k \beta_{4} \lambda_{1}}{\beta_{1}\left(\exp \left(\lambda_{1}\left(\xi+\xi_{0}\right)\right)-1\right)}\right), \\
k & >l, \quad \beta_{3}>0, \quad \beta_{2}<0 .
\end{aligned}
$$

Case 3. $\lambda_{1}^{2}-4 \mu_{1}=0, \mu_{1} \neq 0, \lambda_{1} \neq 0$,

$$
\begin{aligned}
V_{8} & =\frac{\beta_{4} \lambda_{1} k^{2}+\omega}{\beta_{1} k}-\left(\frac{k \beta_{4} \lambda_{1}^{2}\left(\xi+\xi_{0}\right)}{\beta_{1}\left(2 \lambda_{1}\left(\xi+\xi_{0}\right)+2\right)}\right), \\
k & >l, \quad \beta_{3}>0, \quad \beta_{2}<0 .
\end{aligned}
$$

Case 4. $\lambda_{1}^{2}-4 \mu_{1}=0, \mu_{1}=0, \lambda_{1}=0$,

$$
\begin{gathered}
V_{9}=\frac{\beta_{4} \lambda_{1} k^{2}+\omega}{\beta_{1} k}+\frac{2 k \beta_{4}}{\beta_{1}\left(\xi+\xi_{0}\right)}, \\
k>l, \quad \beta_{3}>0, \quad \beta_{2}<0 .
\end{gathered}
$$

Case 5. $\lambda_{1}^{2}-4 \mu_{1}<0$,

$$
\begin{aligned}
V_{10} & =\frac{\beta_{4} \lambda_{1} k^{2}+\omega}{\beta_{1} k} \\
& +\frac{4 k \beta_{4} \mu}{\beta_{1}\left(\sqrt{4 \mu_{1}-\lambda_{1}^{2}} \tan \left(\frac{\sqrt{4 \mu_{1}-\lambda_{1}^{2}}}{2}\left(\xi+\xi_{0}\right)\right)-\lambda_{1}\right)}, \\
& k>l, \quad \beta_{3}>0, \quad \beta_{2}<0 .
\end{aligned}
$$




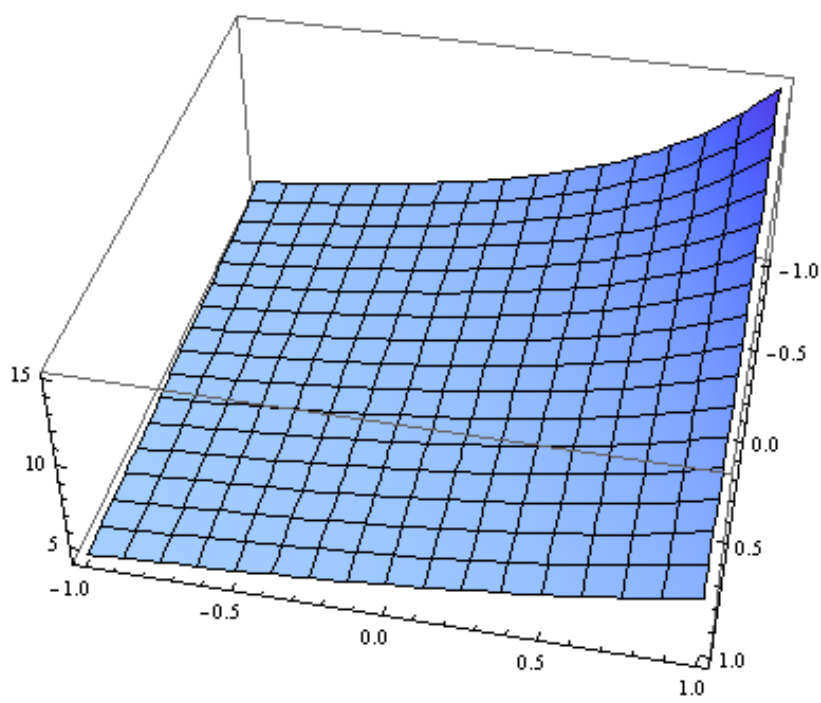

(a)

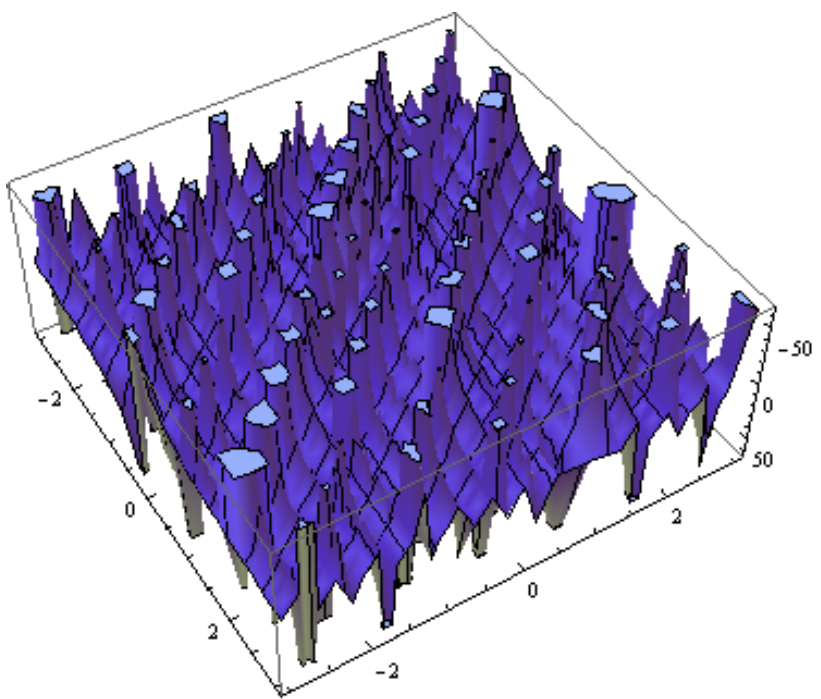

(b)

Figure 2: Exact solitary wave solutions (30) on (a), (31) at (b) with: $\beta_{1}=0.7, \beta_{2}=-1.0, \beta_{3}=1.4, \beta_{4}=-3, k=1.00, l=-0.5, \omega=0.6$ and $\beta_{1}=4, \beta_{2}=-1, \beta_{3}=3, \beta_{4}=3, \lambda_{1}=-1, k=-5.1, \mu_{1}=2, l=0.5, \omega=-0.5, \epsilon=0.5$ respectively.

\section{3 (2+1)-dimensional AKNS equation}

Let the generalized form in [26, 27]

$$
4 v_{x t}+v_{x x x t}+8 v_{x} v_{x y}+4 v_{x x} v_{y}-\gamma v_{x x}=0
$$

Consider,

$$
v(x, y, t)=V, \quad \xi=x+y+k t
$$

Putting (33) in (32), we obtaine

$$
(4 k-\gamma) V^{\prime}+6 V^{\prime 2}+k V^{\prime \prime \prime}=0
$$

Let (34) has solution form (14), after solving we have:

$$
A_{0}=A_{0}, \quad A_{1}=\frac{\gamma}{\lambda_{1}^{2}-4 \mu_{1}+4}, \quad k=\frac{\gamma}{\lambda_{1}^{2}+4-4 \mu_{1}}
$$

Hence, (14) becomes as:

$$
V=A_{0}+\frac{\gamma}{\lambda_{1}^{2}-4 \mu_{1}+4} \exp (-\varphi(\xi))
$$

Case 1. $\lambda_{1}^{2}-4 \mu_{1}>0, \mu_{1} \neq 0$

$$
\begin{aligned}
& V_{11}=A_{0} \\
& +\frac{2 \gamma \mu_{1}}{\left(\lambda_{1}^{2}-4 \mu_{1}+4\right)\left(-\sqrt{\lambda_{1}^{2}-4 \mu_{1}} \tanh \left(\frac{\sqrt{\lambda_{1}^{2}-4 \mu_{1}}}{2}\left(\xi+\xi_{0}\right)\right)-\lambda_{1}\right)}
\end{aligned}
$$

Case 2. $\lambda_{1}^{2}-4 \mu_{1}>0, \mu_{1}=0$,

$$
V_{12}=A_{0}+\frac{1}{\left(\lambda_{1}^{2}-4 \mu_{1}+4\right)}\left(\frac{\gamma \lambda_{1}}{\exp \left(\lambda_{1}\left(\xi+\xi_{0}\right)\right)-1}\right)
$$

Case 3. $\lambda 1^{2}-4 \mu_{1}=0, \mu_{1} \neq 0, \lambda_{1} \neq 0$,

$$
V_{13}=A_{0}-\frac{1}{\left(\lambda_{1}^{2}-4 \mu_{1}+4\right)}\left(\frac{\gamma \lambda_{1}^{2}\left(\xi+\xi_{0}\right)}{\left(2 \lambda_{1}\left(\xi+\xi_{0}\right)+2\right)}\right)
$$

Case 4. $\lambda_{1}^{2}-4 \mu_{1}=0, \mu_{1}=0, \lambda_{1}=0$,

$$
V_{14}=A_{0}+\frac{\gamma}{\left(\lambda_{1}^{2}-4 \mu_{1}+4\right)\left(\xi+\xi_{0}\right)}
$$

Case 5. $\lambda_{1}^{2}-4 \mu_{1}<0$,

$$
\begin{aligned}
& V_{15}=A_{0} \\
& \left(\lambda_{1}^{2}-4 \mu_{1}+4\right)\left(\sqrt{4 \mu_{1}-\lambda_{1}^{2}} \tan \left(\frac{\sqrt{4 \mu_{1}-\lambda_{1}^{2}}}{2}\left(\xi+\xi_{0}\right)\right)-\lambda_{1}\right)
\end{aligned}
$$

\subsection{Unstable nonlinear Schrödinger equation}

The general form of unstable Schrödinger equation[28],

$$
i u_{t}+u_{x x}+2 \eta|u|^{2} u-2 \gamma u=0,
$$

Consider,

$$
u(x, t)=V(\xi) e^{i \delta}, \quad \xi=k x+\omega t, \delta=\alpha x+\beta t
$$

Put (43) in (42),

$$
k^{2} V^{\prime \prime}-\left(\alpha^{2}+\beta+2 \gamma\right) V+2 \eta V^{3}=0, \quad \omega=-2 \alpha k
$$

Let (44) has solution form:

$$
V=A_{0}+A_{1} \exp (-\varphi(\xi))+A_{2}\left(\exp (-\varphi(\xi))^{2}\right.
$$


$a_{0}, a_{1}$ and $a_{2}$ are constants, which can be determined latter. Substituting (45) with (5) in (44), after solving we obtain:

$$
\begin{aligned}
& A_{0}=-\sqrt{\frac{\alpha^{2}+\beta+2 \gamma \lambda_{1}}{2 \eta\left(\lambda_{1}^{2}-4 \mu_{1}\right)}}, \quad A_{1}=-\sqrt{\frac{2\left(\alpha^{2}+\beta+2 \gamma\right)}{\eta\left(\lambda_{1}^{2}-4 \mu_{1}\right)}}, \\
& A_{2}=0, \quad \omega=2 \alpha \sqrt{\frac{-2\left(\alpha^{2}+\beta+2 \gamma\right)}{\left(\lambda_{1}^{2}-4 \mu_{1}\right)}}
\end{aligned}
$$

we have demonstrated possible solutions regarding to (46).

Case I. $\lambda_{1}^{2}-4 \mu_{1}>0, \mu_{1} \neq 0$

$$
\begin{aligned}
& V_{16}=\left(-\sqrt{\frac{\alpha^{2}+\beta+2 \gamma \lambda_{1}}{2 \eta\left(\lambda_{1}^{2}-4 \mu_{1}\right)}}\right. \\
& -\sqrt{\frac{2\left(\alpha^{2}+\beta+2 \gamma\right)}{\eta\left(\lambda_{1}^{2}-4 \mu_{1}\right)}} \\
& \left.\frac{2 \mu_{1}}{\left(-\sqrt{\lambda_{1}^{2}-4 \mu_{1}} \tanh \left(\frac{\sqrt{\lambda_{1}^{2}-4 \mu_{1}}}{2}\left(\xi+\epsilon_{0}\right)\right)-\lambda_{1}\right)}\right) e^{i \delta}
\end{aligned}
$$

Case II. $\lambda_{1}^{2}-4 \mu_{1}>0, \mu_{1}=0$,

$$
\begin{aligned}
V_{17} & =\left(-\sqrt{\frac{\alpha^{2}+\beta+2 \gamma \lambda}{2 \eta\left(\lambda^{2}-4 \mu\right)}}\right. \\
& \left.-\sqrt{\frac{2\left(\alpha^{2}+\beta+2 \gamma\right)}{\eta\left(\lambda^{2}-4 \mu\right)}}\left(\frac{\lambda}{\left(\exp \left(\lambda\left(\xi+\epsilon_{0}\right)\right)-1\right)}\right)\right) e^{i \delta}
\end{aligned}
$$

Case III. $\lambda_{1}^{2}-4 \mu_{1}<0$,

$$
\begin{aligned}
V_{18} & =\left(-\sqrt{\frac{\alpha^{2}+\beta+2 \gamma \lambda_{1}}{2 \eta\left(\lambda_{1}^{2}-4 \mu_{1}\right)}}-\sqrt{\frac{2\left(\alpha^{2}+\beta+2 \gamma\right)}{\eta\left(\lambda_{1}^{2}-4 \mu_{1}\right)}}\right. \\
& \left.\left(\frac{2 \mu_{1}}{\left(\sqrt{4 \mu_{1}-\lambda_{1}^{2}} \tan \left(\frac{\sqrt{4 \mu_{1}-\lambda_{1}^{2}}}{2}\left(\xi+\epsilon_{0}\right)\right)-\lambda_{1}\right)}\right)\right) e^{i \delta}
\end{aligned}
$$

\subsection{DBM equation}

General form in $[29,34]$,

$$
v_{x t}+a e^{v}+d e^{-2 v}=0,
$$

Consider,

$$
v(x, t)=v(\xi), \quad \xi=k x+c t,
$$

Put (51) in (50),

$$
c V^{\prime \prime}+a e^{v}+d e^{-2 v}=0
$$

Let $V=e^{v}$ substitute it and its derivatives in (52), we obtained:

$$
c k V V^{\prime \prime}-c k V^{\prime 2}+a V^{3}+d=0
$$

Suppose (53) has solution form of (45), after solving we have:

$$
\begin{aligned}
& A_{0}=-\frac{\sqrt[3]{d}\left(\lambda_{1}^{2}+2 \mu_{1}\right)}{\sqrt[3]{a}\left(\lambda_{1}^{2}-4 \mu_{1}\right)} \quad A_{1}=-\frac{6 \sqrt[3]{d} \lambda_{1}}{\sqrt[3]{a}\left(\lambda_{1}^{2}-4 \mu_{1}\right)} \\
& A_{2}=-\frac{6 \sqrt[3]{d}}{\sqrt[3]{a}\left(\lambda_{1}^{2}-4 \mu_{1}\right)}, \quad c=\frac{3 a^{2 / 3} \sqrt[3]{d}}{k\left(\lambda_{1}^{2}-4 \mu\right)}
\end{aligned}
$$

Case I. $\lambda_{1}^{2}-4 \mu_{1}>0, \mu_{1} \neq 0$

$$
\begin{aligned}
& V_{19}=-\frac{\left(\lambda_{1}^{2}+2 \mu_{1}\right) d^{\frac{1}{3}}}{a^{\frac{1}{3}}\left(\lambda_{1}^{2}-4 \mu_{1}\right)} \\
& -\frac{12 \lambda_{1} \mu_{1} d^{\frac{1}{3}}}{a^{\frac{1}{3}}\left(\lambda_{1}^{2}-4 \mu_{1}\right)\left(-\sqrt{\lambda_{1}^{2}-4 \mu_{1}} \tanh \left(\frac{\sqrt{\lambda_{1}^{2}-4 \mu_{1}}}{2}\left(\xi+\epsilon_{0}\right)\right)-\lambda_{1}\right)} \\
& -\frac{24 \mu^{2} d^{\frac{1}{3}}}{a^{\frac{1}{3}}\left(\lambda_{1}^{2}-4 \mu_{1}\right)\left(-\sqrt{\lambda_{1}^{2}-4 \mu_{1}} \tanh \left(\frac{\sqrt{\lambda_{1}^{2}-4 \mu_{1}}}{2}\left(\xi+\epsilon_{0}\right)\right)-\lambda_{1}\right)^{2}}
\end{aligned}
$$

Case II. $\lambda_{1}^{2}-4 \mu_{1}>0, \mu_{1}=0$,

$V_{20}=$

$-\frac{d^{\frac{1}{3}}}{a^{\frac{1}{3}}}\left(1+\frac{6}{\left(\exp \left(\lambda_{1}\left(\xi+\epsilon_{0}\right)\right)-1\right)}+\frac{6}{\left(\exp \left(\lambda_{1}\left(\xi+\epsilon_{0}\right)\right)-1\right)^{2}}\right)$

Case III. $\lambda_{1}^{2}-4 \mu_{1}<0$,

$$
\begin{aligned}
& V_{21}=-\frac{\left(\lambda_{1}^{2}+2 \mu_{1}\right) d^{\frac{1}{3}}}{a^{\frac{1}{3}}\left(\lambda_{1}^{2}-4 \mu_{1}\right)} \\
& -\frac{12 \lambda_{1} \mu_{1} d^{\frac{1}{3}}}{a^{\frac{1}{3}}\left(\lambda_{1}^{2}-4 \mu_{1}\right)\left(\sqrt{4 \mu_{1}-\lambda_{1}^{2}} \tan \left(\frac{\sqrt{4 \mu_{1}-\lambda_{1}^{2}}}{2}\left(\xi+\epsilon_{0}\right)\right)-\lambda_{1}\right)} \\
& -\frac{24 \mu_{1}^{2} d^{\frac{1}{3}}}{a^{\frac{1}{3}}\left(\lambda_{1}^{2}-4 \mu_{1}\right)\left(\sqrt{4 \mu_{1}-\lambda_{1}^{2}} \tan \left(\frac{\sqrt{4 \mu_{1}-\lambda_{1}^{2}}}{2}\left(\xi+\epsilon_{0}\right)\right)-\lambda_{1}\right)^{2}}
\end{aligned}
$$

\section{Discussion of the results}

We attained that our result in (18) is likely similar to the Eqs. (3.14) and (3.24) in the [23]. It is conversant that our result in (38) is approximately the same as the solution (13) and (19) in [27]. Moreover, solution (47) is nearly equal to solution (17) in [28] and solution (10) in [33]. Furthermore, our constructed solution (57) is likely the same as the solution (3.9) in [34] and solution (3.26b) in [35] respectively. our results are novel and have not been presented in any literature. 


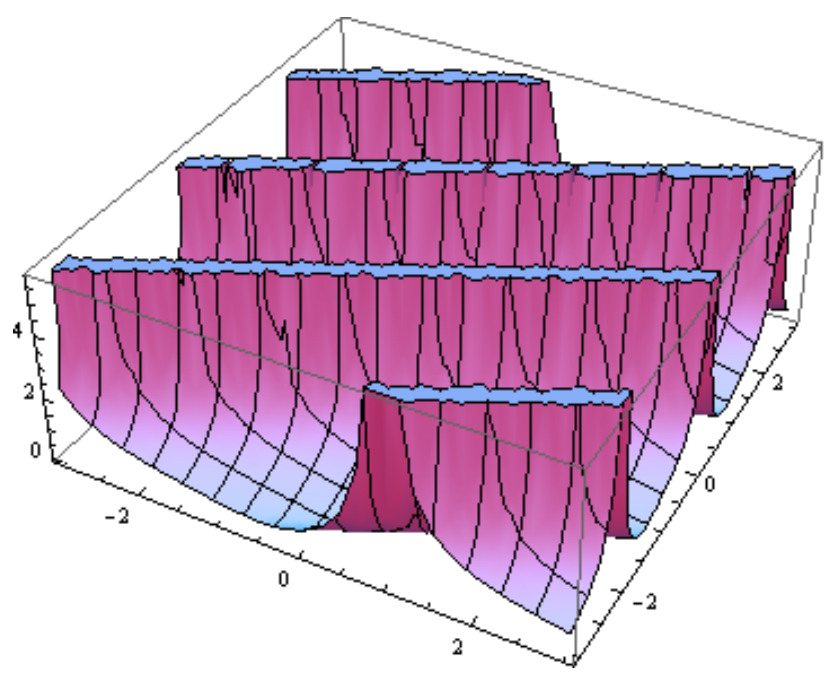

(a)

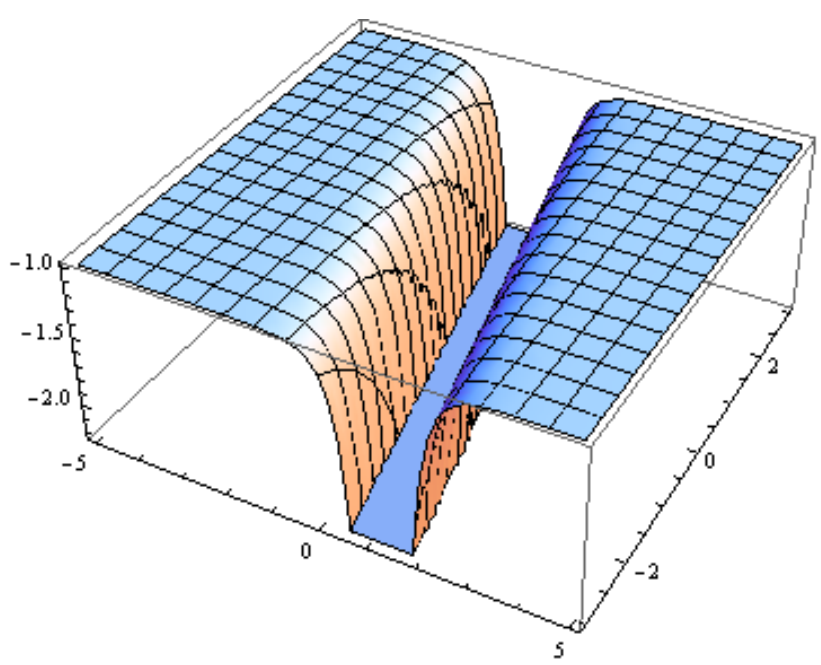

(b)

Figure 3: Graph of (49) at (a), (56) on (b) with: $\varepsilon=0.5, \mu_{1}=4, \lambda_{1}=-2, \beta=-1, \alpha=-1, \gamma=0.5, \eta=1$ and $\varepsilon=-0.5, \mu_{1}=0, \lambda_{1}=1, a=-1$, $d=1, k=1$ respectively.

\section{Conclusion}

We have successfully employed the $\exp (-\varphi(\xi))$-expansion method to construct solutions of important selective waves models. The investigated results have numerous applications in applied sciences and play a fruitful rule in nonlinear sciences. Our technique is simple and straightforward, which is useful for solving different evolutions equations in mathematics and physics.

\section{References}

[1] Ablowitz M.J., Clarkson P.A., Solitons Nonlinear Evolution Equation and Inverse Scattering, 1991, Cambridge University Press, New York.

[2] Fan E., Zhang H., A note on the homogeneous balance method, Phys. Lett. A 1998, 246, 403-406.

[3] Wang1 M., Li X.,Simplified homogeneous balance method and its applications to the Whitham-Broer-Kaup model equations, J. Apply Math Phy, 2014, 2, 823-827.

[4] Seadawy A.R., Modulation instability analysis for the generalized derivative higher order nonlinear Schrödinger equation and its the bright and dark soliton solutions, Journal of Electromagnetic Waves and Applications, 2017, 31, 14, 1353-1362.

[5] Ali A., Seadawy A.R., Lu D., Soliton solutions of the nonlinear Schrödinger equation with the dual power law nonlinearity and resonant nonlinear Schrödinger equation and their modulation instability analysis, Optik, 2017, 145, 79-88.

[6] Lu D., Seadawy A.R., Ali A., Applications of exact traveling wave solutions of Modified Liouville and the Symmetric Regularized Long Wave equations via two new techniques, Results in Physics, 2018, 9, 1403-1410.

[7] Lu D., Seadawy A.R., Arshad M., Applications of extended simple equation method on unstable nonlinear Schrödinger equations, Optik, 2017, 140, 136-144.

[8] Seadawy A.R., Exact solutions of a two-dimensional nonlinear Schrodinger equation, Appl. Math. Lett. 2012, 25, 687-691.

[9] Lu D., Seadawy A.R., Ali A., Dispersive traveling wave solutions of the Equal-Width and Modified Equal-Width equations via mathematical methods and its applications, Results in Physics 2018, 9, 313-320.

[10] Arshad M., Seadawy A.R., Lu D., Wang J., Travelling wave solutions of Drinfeld-Sokolov-Wilson, Whitham-Broer-Kaup and $(2+1)$-dimensionalBroer-Kaup-Kupershmit equations and their applications, Chin. J. Phys. 2017, 55, 780-797.

[11] K A Touchent K.A., Belgacem F.B., Nonlinear fractional partial differential equations systems solutions through a hybrid homotopy perturbation Sumudu transform method, Nonlinear Studies, 2015, 22, 4, 591-600.

[12] Alam M.N., Hafez M.G., Belgace F.B., Applications of the novel ( $G$ ?/G) expansion method to find new exact traveling wave solutions of the nonlinear coupled Higgs field equation, Nonlinear Studies, 2015, 22, 4, 613-633.

[13] Alam M.N., Belgace F.B., Analytical treatment of the evolutionary $(1+1)$ dimensional combined $\mathrm{KdV}-\mathrm{mKdV}$ equation via novel (G/G)-expansion method, Journal of Applied Mathematics and Physics, (2015) 1571-1579.

[14] Khan M.A., Akbar M.A., Belgacem F.B.M., Solitary Wave Solutions for the Boussinesq and Fisher Equations by the Modified Simple Equation Method, Mathematics Letters, 2016, 2, 1, 1-18.

[15] Alam M.N., Belgace F.B., New generalized (G'/G)-expansion method Applications to coupled Konno-Oono and right-handed noncommutative Burgers equations, Advances in Pure Mathematics APM - 2016, 6, 3, 5301012, 168-179.

[16] Alam M.N., Belgace F.B., Exact Traveling Wave Solutions for the (1+1)-Dim Compound KdVB Equation by the Novel (G'/G)Expansion Method, International Journal of Modern Nonlinear 
Theory and Application, 2016, 5, 1, 28-39.

[17] Davy J. Data Modeling and Simulation Applied to Radar Signal Recognition. Prov. Med. Surg. J. 2005, 26, 165-173.

[18] Guariglia E., Entropy and Fractal Antennas, Entropy 2016, 18,3, 84.

[19] Carpinteri A., Cornetti P., A fractional calculus approach to the description of stress and strain localization in fractal media, Chaos Soliton. Fract. 2002, 13, (1), 85-94.

[20] Guariglia E., and Silvestrov S., Fractional-Wavelet Analysis of Positive definite Distributions and Wavelets on D'(C), in Engineering Mathematics II, Silvestrov, Rancic (Eds.), Springer, 2017, 337-353.

[21] Tan B.K., Wu R.S., Nonlinear Rossby waves and their interactions. I. Collision of envelope solitary Rossby waves, Sci. China B 1993, 36, 1367.

[22] Tang X.Y., Shukla P.K., Lie symmetry analysis of the quantum Zakharov equations, Phys. Scr. A 2007, 76, 665-668.

[23] Tariq T., Seadawy A.R., Bistable bright-dark soliary wave solutions of the (3+1)-dimensional Breaking soliton, Boussinesq equation with dual disperion and modified korteweg-de vires kadomstev-petviashvili equations and their applications, Result in physics 2017, 7, 1143-1149.

[24] Seadawy A.R., Nonlinear wave solutions of the threedimensional Zakharov- Kuznetsov-Burgers equation in dusty plasma. Physica A 2015, 439, 124-131.

[25] Abdullah, Seadawy A.R., Wang J., Mathematical methods and solitary wave solutions of three-dimensional ZakharovKuznetsov-Burgers equation in dusty plasma and its applications, Results in Physics 2017, 7, 4269-4277.

[26] Ali A., Seadawy A.R., Lu D., Computational methods and traveling wave solutions for the fourth-order nonlinear AblowitzKaup-Newell-Segur water wave dynamical equation via two methods and its applications, Open Phys. 2018; 16, 219-226.

[27] Helal M.A., Seadawy A.R., Zekry M.H., Stability analysis solutions for the fourth-Order nonlinear ablowitz-kaup-newell-segur water wave equation, Applied Mathematical Sciences, 2013, 7, 3355-3365.

[28] Lu D., Seadawy A.R., Arshad M., Bright-dark solitary wave and elliptic function solutions of unstable nonlinear Schrödinger equation and their applications, Opt Quant Electron 2018, 50, 23.
[29] Seadawy A.R, Ion acoustic solitary wave solutions of twodimensional nonlinear Kadomtsev-Petviashvili-Burgers equation in quantum plasma, Mathematical methods and applied Sciences, 2017, 40, (5), 1598-1607.

[30] Kochanov M.B., Kudryashov N.A., Sinelshchikov D.I., Nonlinar waves on shallow water under an ice cover,higher order expansion, J Apply Math Mech 2013, 77, 25-32.

[31] Seadawy A.R., Stability analysis for Zakharokuznestov equation of weakly nonlinear ion acoustic waves in a plasma, Comput Math Appl 2014, 67, (1), 172-180.

[32] Seadawy A.R., Stability analysis for two dimensional ionacoustic waves in quantum plasmas,Phys plasmas 2014, 21, (5), 052107.

[33] Arshad M., Seadawy A.R., Lu D., WANG J., Optical soliton solutions of unstable nonlinear Schrdinger dynamical equation and stability analysis with applications, Optik, 2018, 157, 597-605.

[34] Seadawy A.R., Lu D., Khater M.A., Bifurcations of traveling wave solutions for Dodd-Bullough-Mikhailov equation and coupled Higgs equation and their applications, Chinese Journal of Physics 2017, 55 1310-1318.

[35] Bahrami B.S., Abdollah H.Z., Exact traveling solutions for some nonlinear physical models by (G'/G)- expansion method,Journal of physics, 2011, 77, 2, 263-275.

[36] Ali A., Seadawy A.R, Lu D., New solitary wave solutions of some nonlinear models and their applications, Advances in Difference Equations, 2018 2018, 232.

[37] Khater A.H., Callebaut D.K., Helal M.A. and Seadawy A.R., Variational Method for the Nonlinear Dynamics of an Elliptic Magnetic Stagnation Line, The European Physical Journal D, 2006, 39, 237-245.

[38] Seadawy A.R., Travelling wave solutions of a weakly nonlinear two-dimensional higher order Kadomtsev-Petviashvili dynamical equation for dispersive shallow water waves, Eur. Phys. J. Plus 2017, 132, 29.

[39] Seadawy A.R., The generalized nonlinear higher order of KdV equations from the higher order nonlinear Schrodinger equation and its solutions. Optik - Int J Light Electron Optics 2017, 139, 31-43.

[40] Seadawy A.R., El-Rashidy K., Rayleigh-Taylor instability of the cylindrical ow with mass and heat transfer, Pramana J. Phys., 2016, 87, 20. 